\title{
Characterization of pentraxin 3 in the horse and its expression in airways
}

\author{
Eve Ramery*, Laurence Fievez, Audrey Fraipont, Fabrice Bureau, \\ Pierre LEKEUX
}

\begin{abstract}
Department for Functional Sciences, Faculty of Veterinary Medicine, University of Liege, Bvd de Colonster, 20, B-4000 Liege, Belgium
\end{abstract}

(Received 5 May 2009; accepted 28 October 2009)

\begin{abstract}
The long pentraxin 3 (PTX3) plays an important role in host defence and its over-expression may contribute to airway injury. The aim of the present study was therefore to characterize in more detail PTX3 and its expression in the horses' airway. Six healthy horses and six horses affected by recurrent airway obstruction (R.A.O.) were submitted to a dusty environment challenge. PTX3 DNA and cDNA were cloned and sequenced. PTX3 expression was evaluated by RT-qPCR, Western blotting and immunohistochemistry in bronchoalveolar lavage fluid (BALF) cells, BALF supernatant and bronchial epithelial cells. An alternative splicing of the second exon of PTX3 occurred, resulting in two forms of the protein: "spliced" (32 kDa) and "full length" (42 kDa). PTX3 was detected in BALF macrophages, neutrophils and bronchial epithelial cells. It was over-expressed in the BALF supernatant from R.A.O.-affected horses in crisis. However, dust was unable to induce PTX3 in BALF cells ex vivo, indicating that dust is an indirect inducer of PTX3. Dust exposure in-vivo induced PTX3 in BALF macrophages but there was no significant difference between healthy and R.A.O.-affected horses. Conversely, PTX3 was over-expressed in the bronchial epithelial cells from R.A.O.-affected horses in crisis. These data indicate a differential regulatory mechanism in inflammatory and bronchial epithelial cells and offer therapeutically interesting perspectives.
\end{abstract}

\section{PTX3 / horse / airway / inflammation}

\section{INTRODUCTION}

The respiratory tract is continuously challenged with potentially infective microorganisms and noxious airborne substances. An effective defense system is necessary for protecting the respiratory tract from pathogen invasion [19]. Airways function not only as a physical barrier but also as a biological sensor for invading microorganisms and cytokines, chemokines and other inflammatory mediators they induce $[15,22]$. These soluble factors, together with infiltrated immune cells, can initiate local inflammatory reactions designed to eliminate the invading

\footnotetext{
* Corresponding author: eve.ramery@ulg.ac.be
}

pathogens, dilute the pathogens' products, limit the damages, and eventually lead to recovery [25]. However, excessive inflammation can induce persistent tissue injury and cause morbidity and mortality [25].

The long pentraxin 3 (PTX3) is a member of the pentraxin family that includes short pentraxins and long pentraxins. Human PTX3 (hPTX3) comprises a C-terminal pentraxin-like domain of 203 amino acids (aa) and a 178 aa N-terminal domain. It has a predicted molecular weight of $40.165 \mathrm{kDa}$. However, in sodium dodecyl sulfate (SDS)-polyacrylamide gel electrophoresis (PAGE) under reducing conditions, the apparent molecular weight is $45 \mathrm{kDa}$. This is due to the presence of an N-glycosylation site in the C-terminal domain [1]. 
On the contrary to short pentraxins (e.g. C-reactive protein, serum amyloid P) that are produced in the liver, PTX3 is produced by diverse cell types, locally, at the site of inflammation. Its production is induced by pathogen recognition. It detects microbial moieties, activates the classical pathway of complement and facilitates recognition by macrophages and dendritic cells (DC) [8]. In studies involving transgenic mice, it was shown that PTX3 over-expression could be protective [5] or deleterious [23], depending on the conditions.

Recurrent airway obstruction (R.A.O.) is a common cause of chronic respiratory disease that occurs in mature horses stabled in a dusty environment containing poor quality hay and straw. It is characterized by episodes of acute airway obstruction (crisis) followed by periods of clinical remission, when horses are kept away from the causative environment. The clinical manifestations of R.A.O. are consequent to lower airway inflammation and involve bronchospasm, excess mucus production and airway hyperresponsiveness to non-specific stimuli [20]. Evidence has accumulated that nucleated cells from bronchoalveolar lavage fluid (BALF) can produce numerous inflammatory mediators, such as interleukin (IL)-8, IL-1 $\beta$ and tumor necrosis factor $\alpha$ (TNF- $\alpha$ ) [9]. Identifying these factors and their regulatory mechanisms will help to understand the role of BALF cells in host defense and inflammatory responses. Using microarray, we recently reported 46 candidate genes with differentially regulated expression between horses suffering from R.A.O. and controls [19]. In particular, the long pentraxin 3 (PTX3) (also called TNF- $\alpha$-stimulated gene 14) was highly over-expressed in BALF cells of horses suffering from R.A.O. and this was confirmed by real time-quantitative polymerase chain reaction (RT-qPCR).

PTX3 plays an important role in host defense and is expressed by a wide range of cell types and tissues. Particularly, over-expression of PTX3 can contribute to airway injury [11]. The aim of the present study was therefore to characterize in more detail PTX3 and its expression in airways. For this purpose, a naturally occurring airway inflammatory disease in horses, namely R.A.O., was used.

\section{MATERIALS AND METHODS}

\subsection{In silico study}

The human complementary desoxyribo-nucleotide acid (cDNA) sequence of PTX3 (Accession number: NM_002852) has been blasted on the equine genome available on $\mathrm{NCBI}^{1}$. Moreover, homologies between human and equine PTX3 genomic sequences have been searched using $\mathrm{UCSC}^{2}$. The equine sequence for PTX3 cDNA obtained in this experiment was blasted on non-human, non-mouse expressed sequence tags (EST) available on NCBI.

\subsection{Primers and antibodies $(\mathrm{Ab})$}

All primers (Tab. I) were designed with Amplify 33 and Oligo6.84 software and purchased from Eurogentec (Seraing, Belgium). The primary antibody, a monoclonal rat anti-human PTX3 (MNB1) was purchased from Alexis (Axxora BVPA, Zandhoven, Belgium). The secondary antibody, a polyclonal rabbit anti-rat immunoglobulin/HRP (No. P0450), was purchased from DakoCytomation (Glostrup, Denmark). A rat IgG2 $\beta$ isotype control (clone 141945) was purchased from R\&D systems (Oxon, UK). The specificity of the monoclonal antibody was tested in total protein extracts from equine peripheral blood leukocytes.

\subsection{Dusty environment challenge}

Six adult half-blood horses diagnosed as affected by R.A.O. ( 4 females and 2 geldings, median; ranges, 14.5 ; 9-22 years) and six considered free from respiratory diseases ( 2 females and 4 geldings, median; ranges, 17.5 ; $11-28$ years) were investigated. The status of the horses (healthy versus affected) was assessed in agreement with the criteria determined by the R.A.O. experts [20], i.e. by history, clinical and endoscopic examination, functional tests (impulse oscillometry, arterial blood gas analysis) and BALF cell counts. Horses were vaccinated and dewormed regularly. The protocol was approved by the Ethics Committee of the University of Liege (Belgium). After a period of 2 months at pasture, all horses were exposed to irritants and aeroallergens by stabling them in the same barn with straw bedding and feeding poor quality, dusty hay for 2 weeks.

\footnotetext{
${ }^{1}$ http://www.ncbi.nlm.nih.gov/

2 http://genome.ucsc.edu/
} 
Table I. Primers.

\begin{tabular}{|c|c|}
\hline Primers s & lence $5^{\prime} \rightarrow 3^{\prime}$ \\
\hline PCR targ & Genomic PTX3 exon 2 \\
\hline Forward & ACACACTGTTTTTCGGAGGTGTC \\
\hline Reverse & CGAGACATCCCAGAAGATAGCC \\
\hline RT-PCR & get/PTX3 $c D N A$ \\
\hline Forward & GACAACGAAATAGACAATGGACTC \\
\hline Reverse & CTTTGTGCCATAGGAAAACAGGAT \\
\hline Forward & TCTGTGGCTTTGACCCAAATGC \\
\hline Reverse & GAAATAGACAATGGACTTCATCC \\
\hline RT-qPCR & $\mathrm{rget} / P T X 3$ \\
\hline Forward & TGGCCAAAACAAAAATAACCAG \\
\hline Reverse & GTGCTTTCAGATGAATGCTGT \\
\hline RT-qPCR & ousekee \\
\hline Forward & СCTTCTCTTGCTGGGTGATTG \\
\hline Reverse & GACAATGAATTTGGCTACAGCA \\
\hline
\end{tabular}

\subsection{BALF macrophage isolation}

BALF was retrieved as previously described and kept at $4{ }^{\circ} \mathrm{C}$ until processed [27]. The cells were collected from BALF by centrifugation for $10 \mathrm{~min}$ at $2000 \mathrm{rpm}$, washed twice and suspended in phosphate buffered saline (PBS) supplemented with $2 \%$ fetal calf serum (FCS). Ten $\mathrm{mL}$ of the mixture were gently layered over $17 \mathrm{~mL}$ of room temperature histopaque-1077 in a $25 \mathrm{~mL}$ centrifuge tube. Tubes were then centrifuged for $10 \mathrm{~min}$ at $1500 \mathrm{rpm}$ and the mononuclear cell layer was removed and washed twice with PBS $+2 \%$ FCS. The cells were then suspended in RPMI 1640-L glutamine supplemented with $10 \%$ heat-inactivated FCS and $1 \%$ penicillinstreptomycin. All cells were seeded in 6-well culture plates and incubated at $37{ }^{\circ} \mathrm{C}$ and $5 \% \mathrm{CO}_{2}$ in a humid atmosphere for $2 \mathrm{~h}$. Non-adherent cells were rinsed out with two washes of PBS $+2 \% \mathrm{FCS}$.

\subsection{BALF cell culture and treatments}

Six culture conditions were applied on BALF cells originating from both healthy and R.A.O.-affected horses in remission: media alone, supplementation with LPS at $10 \mu \mathrm{g} / \mathrm{mL}$, Aspergillus fumigatus at $80 \mathrm{cfu} / \mathrm{mL}$ and hay dust suspensions at $1 \mu \mathrm{g} / \mathrm{mL}, 10 \mu \mathrm{g} / \mathrm{mL}$ and $100 \mu \mathrm{g} / \mathrm{mL}$ respectively. The cells were cultured in RPMI 1640-L glutamine supplemented with 10\% heat-inactivated FCS and $1 \%$ penicillin-streptomycin at $37{ }^{\circ} \mathrm{C}$ and $5 \% \mathrm{CO}_{2}$ for 6 and $24 \mathrm{~h}$. Once the treatment was completed, the supernatants of the culture were collected from each well for measuring PTX3 protein. Cell pellets were immediately lyzed in SDS. All collected samples were stored at $-80^{\circ} \mathrm{C}$ in order to perform Western blotting later.

\subsection{Bronchial tissues from autopsy cases}

Bronchial specimens were obtained within $2 \mathrm{~h}$ after death from 2 horses affected by R.A.O., in severe crisis and euthanized for ethical reasons, and from 3 healthy horses from the slaughterhouse. The specimens were directly mounted in Tissue-Tek ${ }^{\circledR}$ OCT compound and then snap-frozen and stored at $-80{ }^{\circ} \mathrm{C}$. Cryostat tissue sections were performed and used for immuno-histochemistry.

\subsection{DNA isolation, PCR amplification}

Desoxyribo-nucleotide acid (DNA) was extracted from peripheral blood nucleated cells originating from humans and equines, using the QIAamp 96 DNA Blood Kit (Qiagen, Kjvebki, Belgium) and following the manufacturer's instructions.

The PCR product was subjected to electrophoresis on agarose gel. It was then cloned into the cloning vector pGEM $^{\circledR} \mathrm{T}$ (Promega, Leiden, Netherlands). Positive clones were identified by the restriction enzyme FastDigest ${ }^{\circledR}$ MluI (Fermentas, St. Leon-Rot, Germany) and by analyzing the results on agarose gel. The clones were submitted to sequencing.

\subsection{RNA isolation, reverse transcription and PCR amplification}

Equine blood was collected using Paxgene ${ }^{\circledR}$ collection tubes (Sanbion, Uden, Netherlands) and the ribonucleotide acid (RNA) was extracted following the manufacturer's instructions. RNA concentration and purity were measured with spectrophotometer (Nanodrop, ThermoScientific, Wilmington, DE, USA). The cDNA was synthesized and amplified using the First Strand cDNA Synthesis kit for reverse transcription-polymerase chain reaction (RT-PCR) (Roche, Vilvoorde, Belgium) following the manufacturer's instructions. The product was then submitted to sequencing.

\subsection{RT-qPCR}

RT-qPCR was performed as previously described [18]. In brief, RNA was extracted from BALF macrophages using the RNeasy mini kit (Qiagen) and RT-qPCR reactions were performed with IQ Sybr Green Supermix (Bio-Rad, Nazareth, Belgium) 


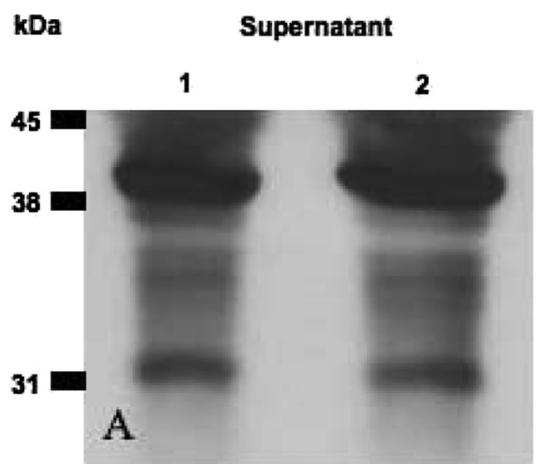

Supernatant

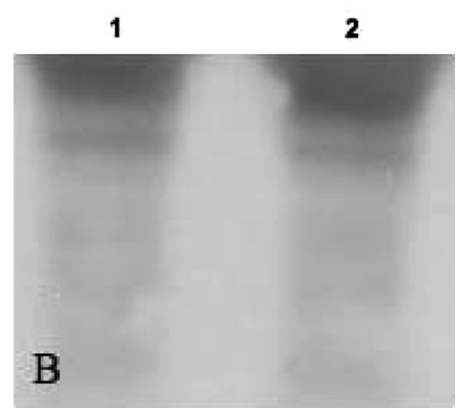

Figure 1. PTX3 specific antibody. (A) Using MNB1 human monoclonal primary antibody (which detects the C-terminal extremity of the PTX3 protein in humans), Western blotting revealed distinct masses for PTX3 protein corresponding to 32 and $42 \mathrm{kDa}$. (B) No band was detected using an IgG2b isotype control. The Western blotting was obtained using BALF cell culture supernatant. The cells originated from R.A.O.-affected horses in remission (named SN1 and SN2) and were challenged for $6 \mathrm{~h}$ with dust suspension $(10 \mu \mathrm{g} / \mathrm{mL})$.

following the manufacturer's instructions. The reaction master mix was prepared as recommended by the manufacturer in a final volume of $20 \mu \mathrm{L}$. Samples were normalized to equine $G A P D H$. The results were analyzed with the ICycler software version 3.OA (Bio-Rad) and a relative quantification of RNA present in the samples was performed using Pfaffl's model and $G A P D H$ gene expression as a reference [17].

\subsection{Western blotting}

Western blotting was performed as previously described [2]. Briefly, cultured BALF cell lysates containing equal amounts of total protein and equal amounts of culture supernatant were heated at $60{ }^{\circ} \mathrm{C}$ with SDS $1 \%$ and bromophenol blue. They were then subjected to SDS-PAGE. Proteins were transferred onto a nitrocellulose membrane. The membrane was blocked with 5\% dry milk and probed with the specific antibodies indicated. The rat $\operatorname{IgG} 2 \beta$ isotype control and first antibody omission were used as negative controls. The blots were visualized with an ECL detection kit.

\subsection{Immuno-histochemistry}

Cytospin preparations of fresh BALF cells were performed. The cells were fixed in periodateparaformaldehyde-lysine (PLP)-sucrose as previously described [24]. Bronchial sections and cytospin preparations were rehydrated in PBS for $5 \mathrm{~min}$ and bronchial sections were treated with acetone for $10 \mathrm{~min}$ and methanol for $10 \mathrm{~min}$. After blocking with $3 \%$ hydrogen peroxide for $20 \mathrm{~min}$ and $5 \%$ of albumin from bovine serum for $30 \mathrm{~min}$, the slides were incubated for $90 \mathrm{~min}$ with either MNB1 monoclonal antibody (1:50 dilution), a rat $\operatorname{IgG} 2 \beta$ isotype control (1:50 dilution), or no $\mathrm{Ab}$, followed by polyclonal rabbit anti-rat immunoglobulins/HRP (1:100 dilution) as secondary Ab. Detection was done by AEC substrate chromogen. Slides were counterstained with Mayer's hematoxylin. For cytological evaluation, cytospins were stained with May-Grumwald Giemsa.

\subsection{Statistical analysis}

Statistical analysis of the healthy and R.A.O.affected groups was performed by Anova using the software Statview 4.01 (SAS Institute, Cary, NC, USA). A $p$ value of less than 0.05 was considered significant.

\section{RESULTS}

\subsection{Characterization of equine $P T X 3$}

The blast identified three homology domains between the human cDNA of PTX3 and the equine genomic sequence of $P T X 3$, localized on horse chromosome 19. From UCSC, we 
identified a gap in the equine genomic sequence corresponding to a part of the exon 2 of the human PTX3.

Our DNA sequencing results confirmed a 120 bp gap in the equine genomic region corresponding to $h P T X 3$ exon 2 . The resulting predictive protein would thus be 340 aa long and weigh $37.282 \mathrm{kDa}$. The cDNA nucleotide sequence we obtained translated into a 235 aa predictive protein weighing $27.153 \mathrm{kDa}$. The Western blotting performed with BALF cell culture medium revealed apparent molecular masses of 32 and $42 \mathrm{kDa}$ (Fig. 1).

Thus the equine PTX3 gene, located on equine chromosome 19 , seems to be organized in three exons separated by two introns. Our results also strongly suggest that an alternative splicing occurs with deletion of the second exon and results in the $32 \mathrm{kDa}$ band on immunoblot.

The two predictive proteins maintain the two cysteine residues and the canonical "pentraxin consensus signature" ( $\mathrm{HxCxS} / \mathrm{TWxS})$. The N-glycosylation Asparagine (Asn) site is also conserved. Alignment analysis of $h P T X 3$ and $e P T X 3$, full length and spliced, aa sequences (Clustal W., European Bioinformatics Institute ${ }^{3}$ ) is shown in Figure 2.

A three-dimensional model for the pentraxin domain in $e P T X 3$ was generated from the highresolution crystal structure of human SAP using Swiss Prot from Expasy.

\subsection{Horse clinical status assessment}

Clinical scores were significantly different between both groups $(p<0.05)$ after dust exposure. The most sensitive value of impulse oscillometry for R.A.O.-affected horse evaluation, $\mathrm{X} 5 \mathrm{~Hz}$ [26] was significantly different between R.A.O.-affected horses $(-0.008 \pm 0.004 \mathrm{kPa} /$ $\mathrm{L} / \mathrm{s})$ and controls $(0.0104 \pm 0.002 \mathrm{kPa} / \mathrm{L} / \mathrm{s})$. The percentage of BALF neutrophils in R.A.O.-affected horses $(22.94 \pm 3.4 \%)$ was significantly increased compared to controls $(12 \pm 4.71 \%)$. The arterial blood oxygen partial pressure was significantly decreased in R.A.O.affected horses $(86.4 \pm 6.9 \mathrm{mmHg})$ when compared to healthy horses $(108.6 \pm 5.9 \mathrm{mmHg})$.

\footnotetext{
3 http://www.ebi.ac.uk/Tools/clustalw2/index.html.
}

\subsection{Cells expressing PTX3 in airways}

Cells able to produce PTX3 in horses' airways were identified by immuno-histochemistry. PTX3 was detected in BALF macrophages and young neutrophils from both R.A.O.-affected and healthy horses after dust exposure. The signal appeared to be stronger in BALF cells from R.A.O.-affected horses. It was almost undetectable in BALF cells from either healthy or R.A.O.-affected horses at pasture (Fig. 3). PTX3 was also detected in eosinophils from a horse suffering from eosinophilia of unknown origin. A strong positive reaction was observed in foamy macrophages.

A positive immuno-histochemical reaction (red) was seen in frozen sections of bronchi from healthy and R.A.O.-affected horses, in bronchial epithelial cells and in infiltrative inflammatory cells (Fig. 4). In bronchial epithelial cells, it localized at the apex of the cells just behind the ciliated process. The staining seemed to be enhanced in horses suffering from R.A.O. No staining was found under negative control conditions.

\subsection{Effect of dust on PTX3 expression}

Following dust exposure, PTX3 was increased in the supernatant originating from horses suffering from R.A.O. (Fig. 5).

Since macrophages showed the strongest positive reaction for anti-PTX3 antibody, we investigated the effect of dust exposure on PTX3 induction in this cell type further. PTX3 mRNA was over-expressed in BALF macrophages following dust exposure (Fig. 6). However, there was no significant difference between R.A.O.-affected horses and healthy horses. Similarly, at the protein level, an enhanced positive reaction for anti-PTX3 antibody was seen in macrophages following dust exposure, in both healthy horses and those suffering from R.A.O., though the reaction was stronger in horses suffering from R.A.O. (Fig. 3).

A. fumigatus and LPS are both known to be present in hay dust, a causative agent of R.A.O., and to induce PTX3 expression. Thus, we studied the involvement of each stimulus on BALF cells ex vivo. The protein was detected in all conditions, including in the control assay. There 
CLUSTAL 2.0.10 multiple sequence alignment

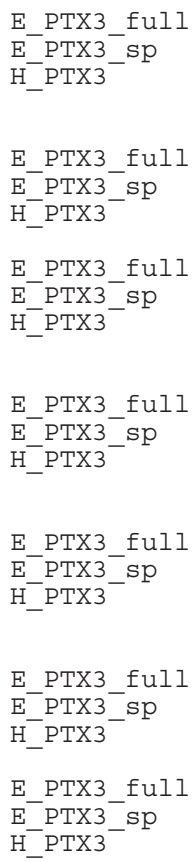

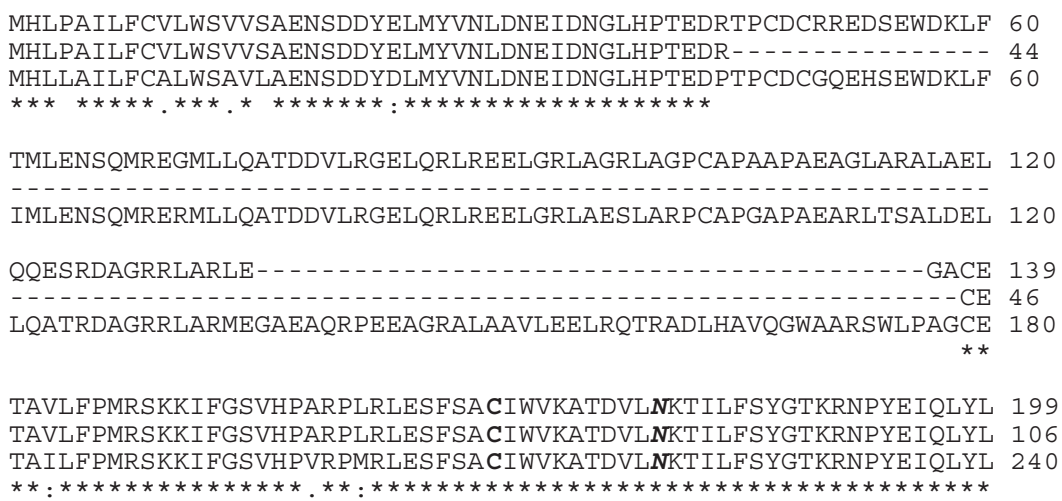

TAVLFPMRSKKIFGSVHPARPLRLESFSACIWVKATDVLNKTILFSYGTKRNPYEIQLYL 199 TAVLFPMRSKKI FGSVHPARPLRLESFSACIWVKATDVLNKTILFSYGTKRNPYEIQLYL 106 TAILFPMRSKKI FGSVHPVRPMRLESFSACIWVKATDVLNKTILFSYGTKRNPYEIQLYL 240

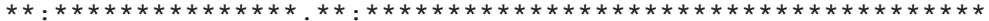

SSQSIVFVVGGEESKLVADTVVSLGRWTHLCSTWNSEKGRTSLWVDGELVASTVEMATGH 259 SSQS IVFVVGGEESKLVADTVVSLGRWT HLCSTWNSEKGRTSLWVDGELVASTVEMATGH 166 SYQS IVFVVGGEENKLVAEAMVSLGRWTHLCGTWNSEEGLTSLWVNGELAATTVEMATGH 300 $* \star * * * * * * * * * * . * * * *::: * * * * * * * * * * . * * * * *: * * * * * *: * * * . *: * * * * * * * *$

VVPEGGILQIGQEKNGCCVGGGFDETLAFSGRLTGFNIWDHVLSNEEIRETGGAESCHIR 319 VVPEGGILQIGQEKNGCCVGGGFDETLAFSGRLTGFNIWDHVLSNEEIRETGGAESCHIR 226 IVPEGGILQIGQEKNGCCVGGGFDETLAFSGRLTGFNIWDSVLSNEEIRETGGAESCHIR 360

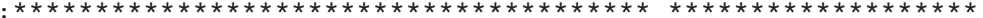

GNVVGWGVTEIQPHGGAQYVS - 340 GNVVGWGVTEIQPHGGAQYVS - 247 GNIVGWGVTEIQPHGGAQYVS - 381 $* *: * * * * * * * * * * * * * * * * * *$

Figure 2. Alignment analysis of amino acid (aa) residues for equine PTX3 full length (E_PTX3_full), spliced (E_PTX3_sp) and human PTX3 (ptx3_H). *Residues in that column are identical in all sequences in the alignment. (:) Conserved substitutions are observed. (.) Semi-conserved substitutions are observed. The pentraxin canonical signature $(\mathrm{H} \times \mathrm{C} \times \mathrm{S} / \mathrm{TW} \times \mathrm{S} / \mathrm{T})$ is underlined and the two cysteines conserved in all the members of the pentraxin family are in bold. The N-glycosylation Asn site is in bold italics.

was no significant variation between the different conditions, neither in cell lysates nor in culture supernatant (results not shown).

\section{DISCUSSION}

The equine $P T X 3$ gene, located on equine chromosome 19 seems to be organized in three exons separated by two introns. The two bands detected by Western blot, at 32 and $42 \mathrm{kDa}$ strongly suggest an alternative splicing. This result is supported by the fact that our cDNA sequence obtained for $P T X 3$ aligned perfectly with Equus caballus cDNA clone LeukoN4 5 F08 A026 5' (accession number: CD464905). To the best of our knowledge, it is the first time PTX3 alternative splicing is reported. Whether this event is due to the $120 \mathrm{bp}$ deletion in the N-terminal domain, and is thus specific to the horse, or occurs in all species remains to be determined.

The C-terminal pentraxin-like domain of equine PTX3 protein is well conserved. It shares $94 \%$ of conserved aa and a similar predicted three-dimensional model with the human protein [14]. This argues for a conserved function of this domain between species.

The C-terminal extremity is implicated in the interaction with $\mathrm{C} 1 \mathrm{q}$ and the subsequent activation of the complement [13]. The antiangiogenic function of the protein is related to its N-terminal extremity [3]. Moreover, binding of PTX3 to Factor $\mathrm{H}$ depends on interactions with both domains [4]. Thus, the alternative forms of the protein observed in the horse 


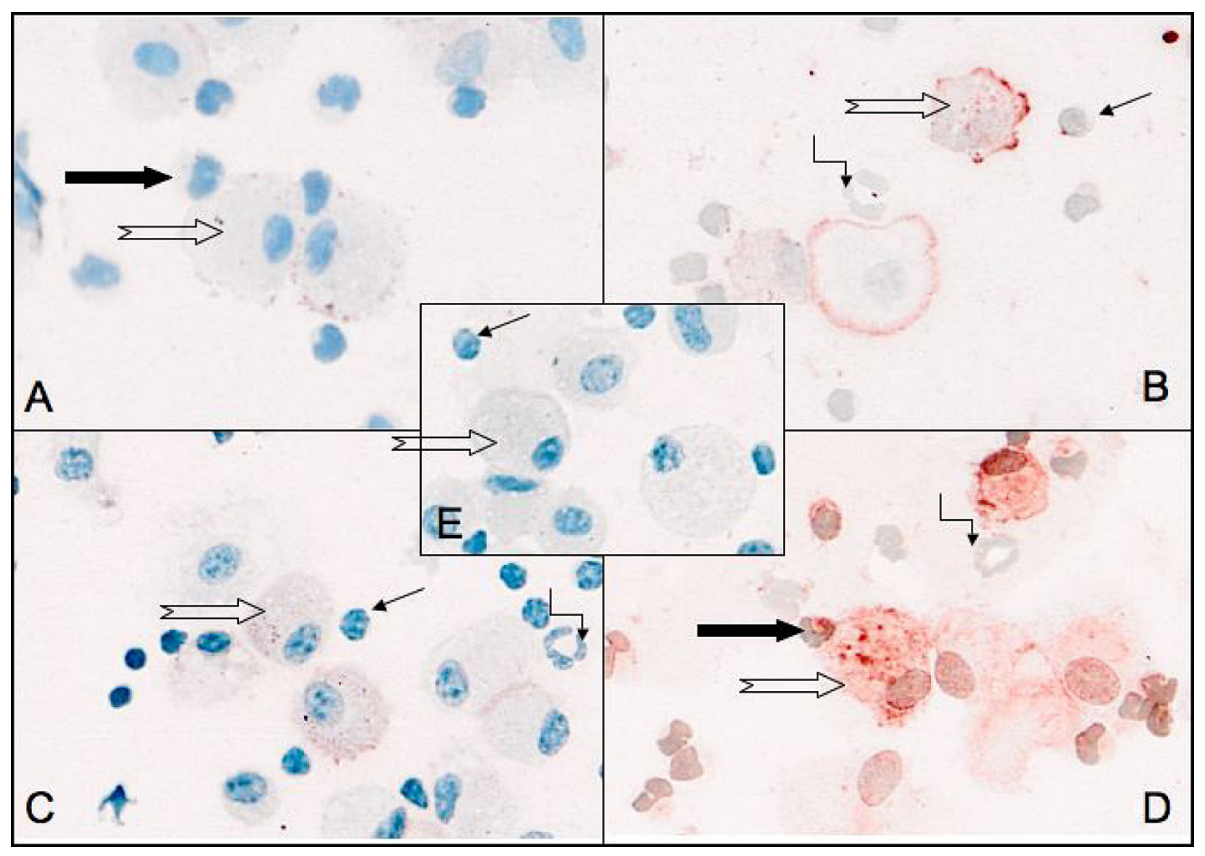

Figure 3. PTX3 expression in BALF cells. Large straight black arrows indicate young neutrophiles, empty arrows indicate macrophages, straight fine black arrows indicate lymphocytes and angled black arrows indicate mature neutrophiles. PTX3 immuno-staining appears in red. (A) BALF cells from a healthy horse at pasture. (B) BALF cells from a healthy horse after two weeks of dust exposure. (C) BALF cells from an R.A.O.-affected horse at pasture. (D) BALF cells from an R.A.O.-affected horse after two weeks of dust exposure. (E) BALF cells from the same horse (R.A.O.-affected horse after two weeks of dust exposure) as in (D) butusing an IgG2B isotype control (control experiment; similar results were obtained by omitting the primary antibody). Magnification $\times 200$. (A color version of this figure is available online at www.vetres.org.)

provide an opportunity to gain better knowledge of the implication of each domain in vivo.

The N-linked glycosylation site on the C-terminal pentraxin-like domain of PTX3 is conserved in the horse. It is probably responsible for the $5 \mathrm{kDa}$ higher molecular weight (32 and $42 \mathrm{kDa}$ instead of 27 and $37 \mathrm{kDa}$ respectively) observed on Western blot under reducing conditions when compared to the predicted molecular weight of the protein [1].

Using immuno-histochemistry, we determined that PTX3 protein is expressed in BALF macrophages, young neutrophils and bronchial epithelial cells in equines.

PTX3 over-expression observed in the BALF supernatant from R.A.O.-affected horses in crisis confirmed previous results obtained in
BALF cells [18]. In BALF cells, macrophages showed the strongest expression of PTX3. In particular, there was a strong expression of PTX3 by dust-activated foamy macrophages. These findings corroborate previous results [21], suggesting that the production of PTX3 is induced when macrophages differentiate into foam cells. Both R.A.O.-affected and healthy horses presented an exacerbation of PTX3 expression in their macrophages following dust exposure. This suggests that a certain degree of inflammation also occurs in healthy horses following dust exposure and that another cell type than macrophages is implicated in the difference in airway PTX3 expression between R.A.O.-affected horses and healthy horses upon dust challenge. 


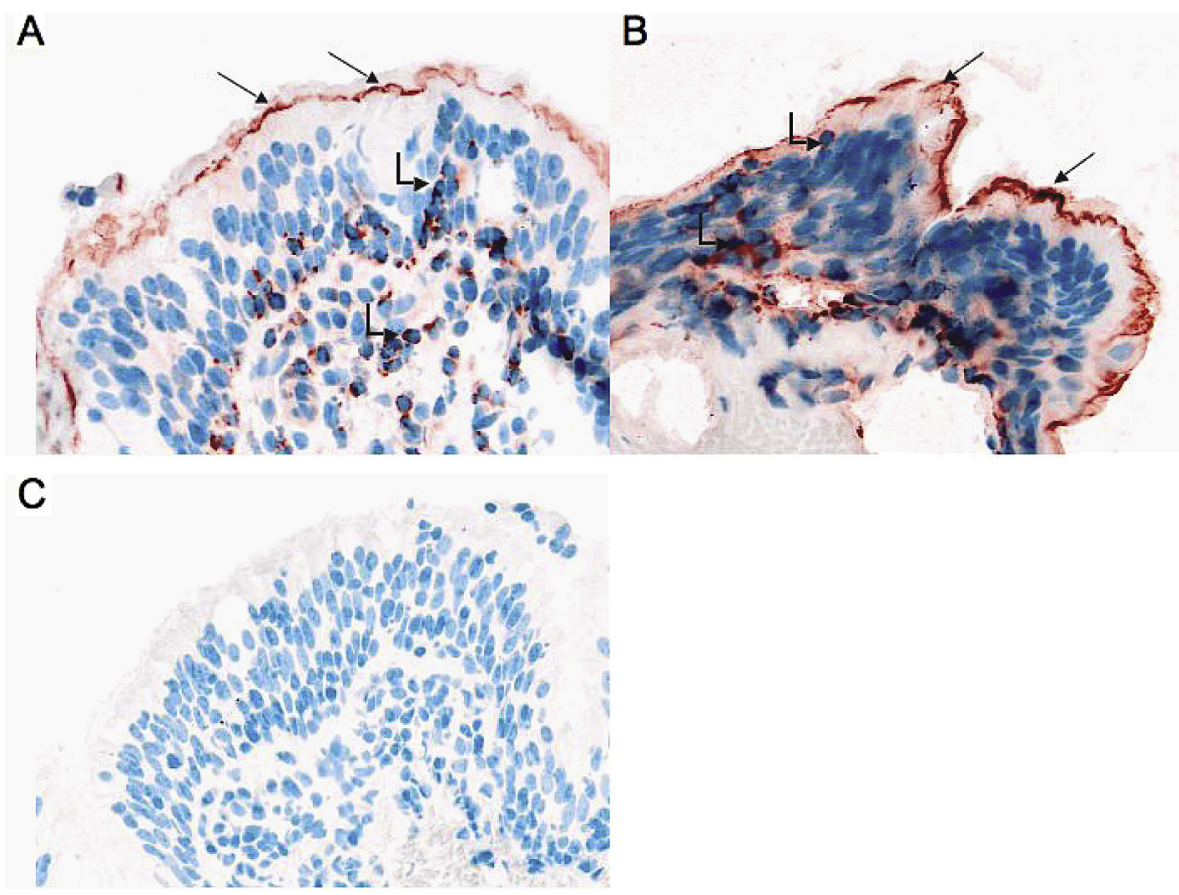

Figure 4. PTX3 expression in tissue samples. PTX3 was constitutively expressed in bronchial epithelial cells (straight arrows) and was localized at the apex just behind ciliated processes. It was also detected in infiltrative inflammatory cells (angled arrows). (A) Bronchial section from a healthy horse. (B) Bronchial section from a horse suffering from R.A.O. with over-expression of PTX3 in bronchial epithelial cells and an increase of the number of infiltrative inflammatory cells expressing PTX3. (C) Control experiment carried out using an IgG2 $\beta$ isotype showed no staining of PTX3 (the same results were obtained by omitting the primary antibody). Magnification $\times 200$. (A color version of this figure is available online at www.vetres.org.)

More horses should be investigated to definitively confirm these results but a stronger PTX3 immunohistologic signal was noticed in the bronchial epithelial cells of horses suffering from R.A.O. in crisis, in comparison with controls. Considering epithelial cells as one of the major cell types in the airways, PTX3 produced at this level could play an important role in the inflammatory process. In lung epithelial cells, PTX3 expression is regulated by a JNK-dependant, NFкB-independent pathway [10]. A comparable mechanism could be involved in bronchial epithelial cells. Such a differential regulatory mechanism between hematopoietic and nonhematopoietic cells is supported by the completely different responses of these two different cell populations to glucocorticoid treatment [6].
This would be of particular interest because PTX3 expression in respiratory epithelial cells could thus be therapeutically targeted.

The fact that LPS, conidia and dust suspension challenges failed to induce PTX3 protein production in BALF cells ex vivo suggests that dust is not a direct inducer of PTX3. To support this finding, PTX3 expression was observed in rat lungs after LPS, hemorrhagic shock/resuscitation and mechanical ventilation but, in vitro, lung epithelial cells did not respond either to LPS or to mechanical stretch stimulation [16]. In addition, silica was shown to induce PTX3 release from monocytes + pneumocytes co-cultures but neither in pneumocytes nor in monocytes alone. Moreover, both silica and IL-1 $\beta$ induced PTX3 expression in co-cultures but 


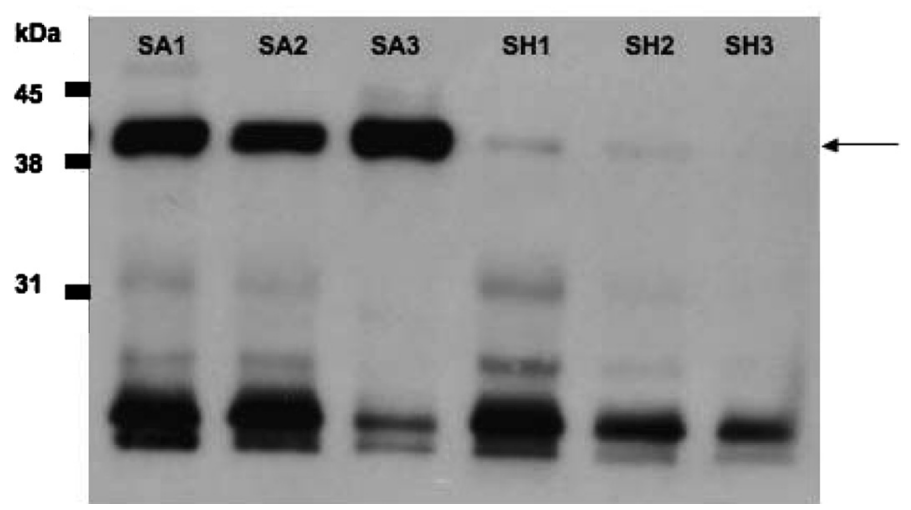

Figure 5. PTX3 in BALF supernatant (10× concentrated) from healthy horses (SH1, SH2, SH3) and R.A.O.-affected horses (SA1, SA2, SA3) after 2 weeks of dust exposure.

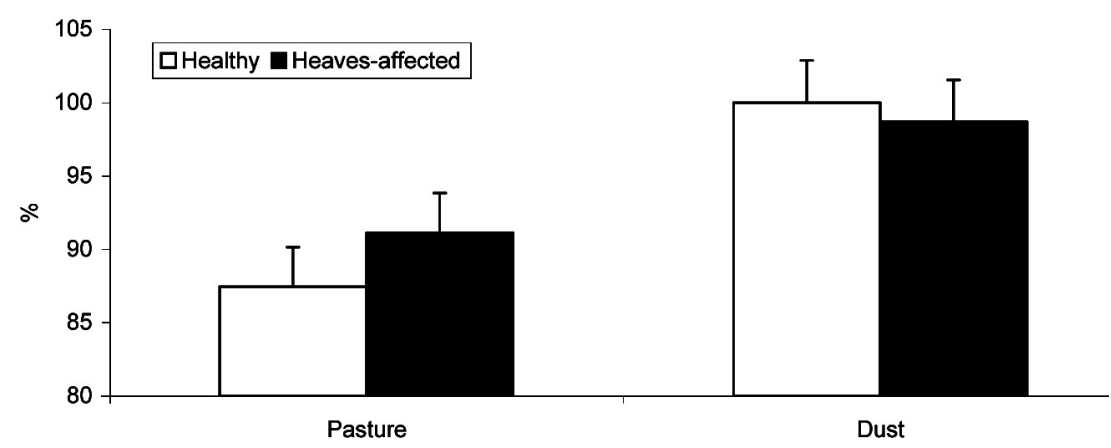

Figure 6. Relative gene expression of $P T X 3$ normalized to GAPDH in BALF macrophages. The results are given in $\%$ of the maximal value.

no difference in PTX3 levels was detected upon combined silica and IL-1 $\beta$ treatment compared to IL-1 $\beta$ [12]. In another study, TNF- $\alpha$ but neither LPS nor mechanical stretch induced PTX3 in A549 lung epithelial cells [7]. A similar effect of TNF $\alpha$ was also shown in BEAS-2B bronchial epithelial cells [10]. TNF- $\alpha$ and IL$1 \beta$ are over-expressed in R.A.O.-affected horses' airways [9]. These cytokines could thus be intermediate mediators for dust-induced PTX3 expression in BALF cells.

We demonstrate that alternative splicing occurs in PTX3 in horses. We also provide further evidence that PTX3 is not directly induced by dust exposure but rather by enhancers of inflammatory mediators such as LPS, TNF- $\alpha$ and IL-1 $\beta$. Finally, the regulatory pathway responsible for the induction of PTX3 in bronchial epithelial cells could be of interest because it could provide a respiratory-specific target for future treatments.

Acknowledgements. The authors thank Drs Michel Georges, Christophe Desmet and Dimitri Pirottin for helpful discussions and scientific advice in molecular biochemistry, Dr Nadine Antoine, Joelle Piret and Monique Henket for their help in immuno-histology and cytology, Dr Tatiana Art for the critical review, Dr Dorine Olejnik and Jean-Clément Bustin for their help with horse management, and Martine Leblond and Ilham Sbai for excellent technical and secretarial assistance. Eve Ramery is a research fellow at the "Fonds de la Formation à la Recherche dans l'Industrie et l'Agriculture", FRIA, Belgium. 


\section{REFERENCES}

[1] Bottazzi B., Vouret-Craviari V., Bastone A., De Gioia L., Matteucci C., Peri G., et al., Multimer formation and ligand recognition by the long pentraxin PTX3. Similarities and differences with the short pentraxins C-reactive protein and serum amyloid P component, J. Biol. Chem. (1997) 272:32817-32823.

[2] Burnette W.N., "Western blotting": electrophoretic transfer of proteins from sodium dodecyl sulfate-polyacrylamide gels to unmodified nitrocellulose and radiographic detection with antibody and radioiodinated protein A, Anal. Biochem. (1981) 112:195-203.

[3] Camozzi M., Rusnati M., Bugatti A., Bottazzi B., Mantovani A., Bastone A., et al., Identification of an antiangiogenic FGF2-binding site in the $\mathrm{N}$ terminus of the soluble pattern recognition receptor PTX3, J. Biol. Chem. (2006) 281:22605-22613.

[4] Deban L., Jarva H., Lehtinen M.J., Bastone A., Jokiranta T.S., Mantovani A., Meri S., Binding of the long pentraxin PTX3 to factor $\mathrm{H}$ : interacting domains and function in the regulation of complement activation, $\mathrm{J}$. Immunol. (2008) 181:8433-8440.

[5] Dias A.A., Goodman A.R., Dos Santos J.L., Gomes R.N., Altmeyer A., Bozza P.T., et al., TSG-14 transgenic mice have improved survival to endotoxemia and to CLPinduced sepsis, J. Leukoc. Biol. (2001) 69:928-936.

[6] Doni A., Mantovani G., Porta C., Tuckermann J., Reichardt H.M., Kleiman A., et al., Cell-specific regulation of PTX3 by glucocorticoid hormones in hematopoietic and nonhematopoietic cells, J. Biol. Chem. (2008) 44:29983-29992.

[7] Dos Santos C.C., Han B., Andrade C.F., Bai X., Uhlig S., Hubmayr R., et al., DNA microarray analysis of gene expression in alveolar epithelial cells in response to TNF- $\alpha$, LPS, and cyclic stretch, Physiol. Genomics (2004) 19:331-342.

[8] Garlanda C., Bottazzi B., Bastone A., Mantovani A., Pentraxins at the crossroads between innate immunity, inflammation, matrix deposition, and female fertility, Annu. Rev. Immunol. (2005) 23:337-366.

[9] Giguere S., Viel L., Lee E., MacKay R.J., Hernandez J., Franchini M., Cytokine induction in pulmonary airways of horses with heaves and effect of therapy with inhaled fluticasone propionate, Vet. Immunol. Immunopathol. (2002) 85:147-158.

[10] Han B., Mura M., Andrade C.F., Okutani D., Lodyga M., dos Santos C.C., et al., TNFalpha-induced long pentraxin PTX3 expression in human lung epithelial cells via JNK, J. Immunol. (2005) 175:8303-8311.

[11] He X., Han B., Liu M., Long pentraxin 3 in pulmonary infection and acute lung injury, Am. J. Physiol. Lung Cell. Mol. Physiol. (2007) 292:1039-1049.

[12] Herseth J.I., Volden V., Schwarze P.E., Låg M., Refsnes M., IL-1beta differently involved in IL-8 and FGF-2 release in crystalline silica-treated lung cell cocultures, Part. Fibre Toxicol. (2008) 13:5-16.
[13] Inforzato A., Peri G., Doni A., Garlanda C., Mantovani A., Bastone A., et al., Structure and function of the long pentraxin PTX3 glycosidic moiety, fine-tuning of the interaction with $\mathrm{C} 1 \mathrm{q}$ and complement activation, Biochemistry (2006) 45:11540-11551.

[14] Inforzato A., Rivieccio V., Morreale A.P., Bastone A., Salustri A., Scarchilli L., et al., Structural characterization of PTX3 disulfide bond network and its multimeric status in cumulus matrix organization, Biol. Chem. (2008) 283:10147-10161.

[15] Kagnoff M.F., Eckmann L., Epithelial cells as sensors for microbial infection, J. Clin. Invest. (1997) 100:6-10.

[16] Okutani D., Han B., Mura M., Waddell T.K., Keshavjee S., Liu M., High-volume ventilation induces pentraxin 3 expression in multiple acute lung injury models in rats, Am. J. Physiol. Lung Cell. Mol. Physiol. (2007) 292:L144-L153.

[17] Pfaffl M.W., A new mathematical model for relative quantification in real-time RT-PCR, Nucleic Acids Res. (2001) 29:2002-2007.

[18] Ramery E., Closset R., Bureau F., Art T., Lekeux P., Relevance of using a human microarray to study gene expression in heaves-affected horses, Vet. J. (2008) 177:216-221.

[19] Reynolds H.Y., Pulmonary host defenses, Alcohol. Clin. Exp. Res. (1995) 19:6-10.

[20] Robinson N.E., Chairperson's introduction: International Workshop on Equine Chronic Airway Disease, Michigan State University, 16-18 June 2000, Equine Vet. J. (2001) 33:5-19.

[21] Savchenko A., Imamura M., Ohashi R., Jiang S., Kawasaki T., Hasegawa G., et al., Expression of pentraxin 3 (PTX3) in human atherosclerotic lesions, J. Pathol. (2008) 215:48-55.

[22] Simon R.H., Paine R., Participation of pulmonary alveolar epithelial cells in lung inflammation, J. Lab. Clin. Med. (1995) 126:108-118.

[23] Souza D.G., Soares A.C., Pinho V., Torloni H., Reis L.F., Teixeira M.M., Dias A.A., Increased mortality and inflammation in tumor necrosis factor-stimulated gene-14 transgenic mice after ischemia and reperfusion injury, Am. J. Pathol. (2002) 160:1755-1765.

[24] St-Laurent J., Boulay M.E., Prince P., Bissonnette E., Boulet L.P., Comparison of cell fixation methods of induced sputum specimens: an immunocytochemical analysis, J. Immunol. Methods (2006) 308:36-42.

[25] Tracey K.J., The inflammatory reflex, Nature (2002) 420:853-859.

[26] Van Erck E., Votion D., Art T., Lekeux P., Qualitative and quantitative evaluation of equine respiratory mechanics by impulse oscillometry, Equine Vet. J. (2006) 38:52-58.

[27] Watson M.L., White A.M., Campbell E.M., Smith A.W., Uddin J., Yoshimura T., Westwick J., Anti-inflammatory actions of interleukin-13: suppression of tumor necrosis factor-alpha and antigen-induced leukocyte accumulation in the guinea pig lung, Am. J. Respir. Cell Mol. Biol. (1999) 20:1007-1012. 